\title{
BMJ Open Risk of harm in synthetic and biological intervention trials in patients with inflammatory arthritis: protocol for a metaepidemiological study focusing on contextual factors
}

\author{
Esben Malm (1) ,,2 Sabrina Mai Nielsen (1) ,1,2 Johannes Berg, ${ }^{1,2}$ \\ John P. A. loannidis (1) , ${ }^{3}$ Daniel Furst, ${ }^{4}$ Josef S. Smolen, ${ }^{5}$ Peter C. Taylor (1) ${ }^{6}$ \\ Lars Erik Kristensen, ${ }^{1}$ Simon Tarp, ${ }^{1}$ Torkell Ellingsen (D) , ${ }^{2}$ Robin Christensen ${ }^{1,2}$
}

To cite: Malm E, Nielsen SM, Berg J, et al. Risk of harm in synthetic and biological intervention trials in patients with inflammatory arthritis: protocol for a metaepidemiological study focusing on contextual factors. BMJ Open 2021;11:e049850. doi:10.1136/ bmjopen-2021-049850

- Prepublication history for this paper is available online To view these files, please visit the journal online (http://dx.doi. org/10.1136/bmjopen-2021 049850).

Received 05 February 2021 Accepted 09 August 2021

Check for updates

(C) Author(s) (or their employer(s)) 2021. Re-use permitted under CC BY-NC. No commercial re-use. See rights and permissions. Published by BMJ.

For numbered affiliations see end of article.

Correspondence to Dr Robin Christensen; robin.christensen@regionh.dk

\section{ABSTRACT}

Introduction Inflammatory arthritis (IA) conditions, including rheumatoid arthritis, psoriatic arthritis and axial spondyloarthritis, are characterised by inflammatory infiltration of the joints. Biological disease-modifying antirheumatic drugs (bDMARDs) and targeted synthetic disease-modifying antirheumatic drugs (tsDMARDs), respectively, reduce the effects of proinflammatory cytokines and immune cells to ameliorate disease. However, immunosuppression can be associated with high rates of serious adverse events (SAEs), including serious infections, and maybe an increased risk of malignancies and cardiovascular events. Currently, there is no empirical evidence on the extent to which contextual factors and risk of bias (RoB) domains may modify these harm signals in randomised trials.

Methods and analysis We will search MEDLINE (via PubMed) for systematic reviews published since April 2015 and all Cochrane reviews. From these reviews, randomised trials will be eligible if they include patients with an IA condition with at least one group randomly allocated to bDMARD and/or tsDMARD treatments. A predefined form will be used for extracting data on population characteristics (eg, baseline characteristics or eligibility criteria, such as medication background) and specific harm outcome measures, such as number of withdrawals, numbers of patients discontinuing due to adverse events and number of patients having SAEs. RoB in individual trials will be assessed using a modified Cochrane RoB tool. We will estimate the potentially causal harm effects related to the experimental intervention compared with control comparator as risk ratios, and heterogeneity across randomised comparisons will be assessed statistically and evaluated as inconsistency using the $\mathrm{I}^{2}$ Index. Our metaregression analyses will designate population and trial characteristics and each RoB domain as independent variables, whereas the three harm domains will serve as dependent variables.

Ethics and dissemination Ethics approval is not required for this study. Results will be disseminated through publication in international peer-reviewed journals. PROSPERO registration number CRD42020171124.
Strengths and limitations of this study

- This will be an extensive and comprehensive risk of harm analysis of biological disease-modifying antirheumatic drugs and targeted synthetic diseasemodifying antirheumatic drugs across multiple inflammatory arthritis diagnoses unlike previous assessments that have been more circumscribed.

- A large array of contextual factors and risk of bias items will be assessed.

- Despite the comprehensive nature of the assessment, the study may be limited by poor reporting (ie, lack of data/information for certain contextual factors and uncommon harms), and some metaregression assessments may be affected by ecological bias due to the use of aggregated data, and metaconfounding.

\section{INTRODUCTION}

Background

Inflammatory arthritis (IA) is a heterogeneous group of autoimmune diseases that includes rheumatoid arthritis (RA), psoriatic arthritis (PsA), axial spondyloarthritis (AxSpA, including ankylosing spondylitis (AS) ). ${ }^{12}$ These diseases are characterised by inflammatory infiltration of the joints, ${ }^{3}$ resulting in pain, swelling, stiffness and restricted movement. ${ }^{4}$ Ultimately, they can have a detrimental impact on quality of life and can cause progressive disability and premature death. ${ }^{56}$ Not only are the implications severe but also the diseases are a global concern; RA alone affects about $1 \%$ of the world's population. ${ }^{7}$

Aside from conventional synthetic diseasemodifying antirheumatic drugs (csDMARDs) including methotrexate (MTX), targeted therapies, consisting of biological 
disease-modifying antirheumatic drugs (bDMARDs) and targeted synthetic disease-modifying antirheumatic drugs (tsDMARDs), are considered effective for treating IA per se. ${ }^{8-10}$ Notably, bDMARDs work by targeting specific molecules or receptors, ${ }^{11}$ such as tumour necrosis factor alpha, which is a potent inflammatory cytokine produced by $\mathrm{T}$ cells and macrophages. ${ }^{12}{ }^{13}$ In contrast, tsDMARDs target intracellular pathways and reduce the effect of cytokines known to drive the proinflammatory machinery of cellular immune response. ${ }^{14-16}$ As such, bDMARDs and tsDMARDs are immunomodulatory; although effective in alleviating symptoms of IA, they also carry risk of harm (defined as 'the totality of possible adverse consequences of an intervention or therapy ${ }^{17}$ ). Indeed, meta-analyses and an observational cohort study have shown that bDMARDs are associated with higher rates of serious infections ${ }^{18-20}$ and potentially dose-dependent increased risk of malignancies. ${ }^{21}$ Some metastudies and systematic literature reviews suggest that tsDMARD rates of serious infections and malignancies are no different from those of bDMARDs. ${ }^{1622} 23$ Recent reviews report a possibly increased risk of venous thromboembolism with tsDMARDs. ${ }^{23} 24$

Randomised controlled trials (RCTs) inevitably vary with respect to eligibility criteria, patient characteristics and internal validity, which may distort their results (ie, the harm signal) and thus potentially bias their interpretation. ${ }^{25} 26$ If evidence were available on which trial characteristics to adjust for when interpreting harm from metaresearch, these covariates would be considered important contextual factors. Currently, a contextual factor is broadly defined as a '...variable that is not an outcome of the study but needs to be recognized (and measured) to understand the study results. This includes potential confounders and effect modifiers' ${ }^{27}$ However, we currently have no empirical evidence concerning the extent to which contextual factors, such as population and trial characteristics and risk of bias (RoB) domains, modify the harm signals from trials testing targeted therapies (ie, bDMARDs and tsDMARDs) across IA diseases.

\section{Rationale}

Designing, conducting and reporting RCTs should incorporate methods-such as concealing randomised allocation, blinding participants and personnel, and appropriately engaging the intention-to-treat population-that avoid biases resulting from incompatibilities between the intervention and control groups. Empirical evidence suggests that the absence of rigorous methodology can lead to biased intervention effect estimates (ie, net benefits) ${ }^{28}$ Contextual factors, such as population and trial characteristics, also have been shown to be possible effect modifiers when assessing benefits. ${ }^{8}{ }^{29}$

The effect on harms of RoB, trial characteristics and contextual factors (eg, population characteristics) has yet to be investigated, and using metaepidemiology is the method of choice for doing so. ${ }^{30}$ Previous metaepidemiological research within rheumatology has primarily investigated the effect of methodological quality (internal validity) on treatment effects (benefit). ${ }^{8293132}$ To our knowledge, no one has yet investigated the importance in IA therapies of contextual factors (ie, population and trial characteristics) and RoB when the outcome is harmful effects.

\section{Aims and objectives}

This exploratory metaepidemiological study aimed to improve harm reporting by investigating the influence of RoB domains, trial characteristics and contextual factors on the three harm measures: all withdrawals (WD), withdrawals due to adverse events (WD d/t AEs) and serious adverse events (SAEs), ${ }^{17}$ as well as mortality among patients with IA who were treated with bDMARDs and/or tsDMARDs. Our objective is to explore whether specific participant, drug classes, and trial characteristics (ie, contextual factors including RoB domains) have a quantitative influence in terms of effect modification and/ or distortion due to biases on the observed likelihood of harm from an experimental intervention compared with control comparators in randomised trials. If contextual factors have such an influence, we hope to shed light on their importance to future trial reporting and interpretation of harm when reporting randomised trials. ${ }^{17}$

\section{Protocol and registration}

This protocol was developed in accordance with the V.1.07 2018 Methodological Expectations for Cochrane Intervention Reviews recommendations of the Cochrane Collaboration $^{33}$ and reported according to the Preferred Reporting Items for Systematic Reviews and Meta-Analyses Extension for Protocols guidelines. ${ }^{34}$ The study protocol was prespecified and registered in PROSPERO on 17 August 2020 (CRD number: CRD42020171124).

\begin{tabular}{ll}
\hline Table 1 Research objective described using PICO \\
\hline Participants & $\begin{array}{l}\text { Adult patients with rheumatoid arthritis, } \\
\text { psoriatic arthritis, axial spondyloarthritis or } \\
\text { ankylosing spondylitis }\end{array}$ \\
Intervention & $\begin{array}{l}\text { Targeted therapies (ie, biological disease- } \\
\text { modifying antirheumatic drugs and targeted } \\
\text { synthetic disease-modifying antirheumatic } \\
\text { drugs) }\end{array}$ \\
Comparison & $\begin{array}{l}\text { Placebo, standard care or waiting list/no } \\
\text { intervention, active comparator and unclear } \\
\text { (ie, comparison described in insufficient } \\
\text { detail to fit within the other categories) }\end{array}$ \\
Outcomes & $\begin{array}{l}\text { Number of withdrawals, number of } \\
\text { withdrawals due to adverse events, number } \\
\text { of serious adverse events and number of } \\
\text { patients who died }\end{array}$ \\
\hline
\end{tabular}

PICO, participants, intervention, comparison and outcomes. 


\section{Box 1 Search strategy}

(arthritis[tiab] OR spondyloarthritis[tiab] OR ankylosing[tiab] OR psoriatic[tiab] OR Spondylarthropathies[tiab] OR rheumatoid[tiab] OR Psoriasis[tiab])

AND

("disease-modifying antirheumatic drugs"[tiab] OR "biological agent*"[tiab] OR biologics*[tiab] OR DMARD[tiab] OR abatacept[tiab] OR adalimumab[tiab] $\mathrm{OR}$ anakinra[tiab] $\mathrm{OR}$ apremilast[tiab] OR baricitinib[tiab] OR certolizumab[tiab] OR etanercept[tiab] OR filgotinib[tiab] OR golimumab[tiab] OR guselkumab[tiab] OR infliximab[tiab] OR ixekizumab[tiab] OR rituximab[tiab] OR sarilumab[tiab] OR secukinumab[tiab] OR tocilizumab[tiab] OR tofacitinib[tiab] OR upadacitinib[tiab] OR ustekinumab[tiab])

AND

(((“Systematic Review”[pt] OR Meta-Analysis[pt] OR meta-analysis[tiab] OR "systematic review"[tiab] OR meta-analys*[pt] OR meta-analys*[ti] OR metaanalys*[ti] OR meta-regress*[tiab] OR metaregress*[tiab]) AND (“2015/04/01"[Date - Publication]: "3000”[Date - Publication])) OR “Cochrane Database Syst Rev" [jour])

\section{METHODS AND ANALYSIS}

This metaepidemiological study combines data from a large representative sample of available trials into a single database by assembling trials already included in published systematic reviews. Generic outcome measures that cover important harm domains, namely, (1) WD, (2) WD d/t AEs and (3) SAEs, ${ }^{17}$ and mortality will constitute the dependent variables in the database. Harm effects in relation to the use of targeted therapies will be analysed to determine whether they are affected by contextual factors among population, drug classes (eg, biologics vs small molecules) and trial characteristics.

\section{Eligibility criteria}

We will search MEDLINE (via PubMed) for systematic reviews (published since April 2015) or Cochrane reviews. From these systematic reviews, included randomised trials will (independent of publication year) be eligible if they fulfil our inclusion criteria (see table 1 for participants, intervention, comparison and outcomes (PICO) framework), with at least one group randomly allocated to bDMARD and/or tsDMARD.

The interventions of interest are targeted therapies (ie, bDMARDs and tsDMARDs) approved by either the European Medicines Agency or the U.S. Food and Drug Administration, to date, treating IA conditions in adult populations; this pharmacological distinction will also be used for separate stratified analyses. These therapies will include bDMARDs: abatacept, adalimumab, anakinra, certolizumab, etanercept, golimumab, guselkumab, infliximab, ixekizumab, rituximab, sarilumab, secukinumab, tocilizumab and ustekinumab, and tsDMARDs: apremilast, baricitinib, filgotinib, tofacitinib and upadacitinib.

Only RCTs included in an existing systematic review of patients with IA (RA, PsA and AxSpA (including AS)) will be considered for eligibility. We will exclude reviews (ie, not look for trials) that have been withdrawn.

\section{Box 2 Population and trial characteristics}

\section{Trial eligibility criteria}

- Minimum and maximum required number of SJCs.

- Minimum and maximum required number of TJCs.

- Minimum and maximum required CRP.

- Minimum and maximum required ESR.

- Minimum and maximum allowed disease duration.

$\rightarrow$ RF.

- Anti-CCP antibody status.

- Anti-CCP2 status.

DMARD history

> csDMARD-naïve.

csDMARD-IR (cSDMARD-IRs).

- bDMARD or tsDMARD-IR (bDMARD or tsDMARD-IRs).

Medication background of DMARDs

- MTX.

- Naïve.

- Not using.

- Discontinued.

- Continued.

- Not reported.

- cSDMARDs other than MTX.

- Naïve.

- Not using.

- Discontinued.

- Continued.

- Not reported.

- bDMARDs or tsDMARDs.

- Naïve.

- Not using.

- Discontinued.

- Continued.

- Not reported.

Aggregate (eg, average/median) patient baseline characteristics

$>$ Age.

- Female (\%).

Disease duration.

- ESR.

$\checkmark$ CRP.

Disease Ativity Score.

RF positive (\%).

- Anti-CCP positive (\%).

- CCP2 positive (\%)

$>$ SJCs.

- TJCs.

- Health Assessment Questionnaire-Disability Index.

- Physician global assessment of disease activity.

- Patient global assessment of disease activity.

- Patient-reported pain on the Visual Analogue Scale of 0-100 mm. bDMARD, biological disease-modifying antirheumatic drug; CCP, cyclic citrullinated peptide; CCP2, cyclic citrullinated peptide antibody 2; CRP, $C$ reactive protein; CsDMARD, conventional synthetic disease-modifying antirheumatic drug; DMARD, disease-modifying antirheumatic drug; $\mathrm{ESR}$, erythrocyte sedimentation rate; IR, inadequate responder; RF, rheumatoid factor; SJC, swollen joint count; TJC, tender joint count; tsDMARD, targeted synthetic disease-modifying antirheumatic drug.

Furthermore, only RCTs from the eligible systematic reviews where the full text is available in English will be included. There will be no restriction on publication year of the individual RCTs. 


\section{Information sources and search strategy}

We will search MEDLINE (via PubMed) for eligible metaanalyses or systematic reviews of trials published since April 2015 and eligible Cochrane reviews using the search algorithm shown in box 1 .

\section{Study selection}

Two independent reviewers (JB and EM, with support from $\mathrm{SMN} / \mathrm{RC}$ ) will screen the systematic reviews based on title and abstract, in accordance with eligibility criteria. The same two reviewers will assess the full systematic review texts for eligibility of the reviews and subsequently select the RCTs from the reviews that are eligible, according to our objectives. We will obtain the full text if at least one of the reviewers considers an RCT to be potentially eligible during the screening process. Disagreements will be resolved by consensus or by consulting a third reviewer (RC). EndNote V.X9.2 software will be used to manage the reviews and RCT records retrieved from the search.

\section{Data collection process}

Two reviewers (JB and EM) will extract data using a predefined, standardised data extraction form, and in case of uncertainty, a third reviewer (SMN or RC) will be consulted. If a trial is included in more than one review, the trial will be registered and counted/included once.

\section{Review level}

From the reviews, we will extract data on review registration number, year of publication, first author's name, number of RCTs eligible for our study, and the condition and intervention studied, according to the review title.

\section{Randomised trial level}

From the trials, we will extract data on the first author's name, publication identification (ID), trial duration, duration until switch (eg, relevant for adaptive trial designs), rescue, early escape or crossover, number of participants in each arm, and treatment given in the active and comparator arms (ie, following the PICO framework). Treatment in the comparator arm will be grouped into the following predefined categories: (1) placebo; (2) standard care or waiting list/no intervention; (3) active comparator; or as an ultimate last option, (4) unclear. Outcome measures and data extraction of trial and participant characteristics are specified as follows and listed in box 2 .

\section{Participant characteristics in individual studies}

Data on the following trial eligibility criteria will be extracted

- Binary indicator (0 or 1$)$ of inflammation scored as 1 with $\geq 1$ of the following criteria: erythrocyte sedimentation rate (ESR) $\geq 28 \mathrm{~mm} /$ hour, CRP level $\geq 0.3 \mathrm{mg}$ / $\mathrm{dL}$ and/or morning stiffness lasting $\geq 45 \mathrm{~min}$.

- Minimum and maximum required number of swollen joint counts (SJCs).

- Minimum and maximum required number of tender joint counts (TJCs).

- Minimum and maximum allowed disease duration.
- Rheumatoid factor (RF) and/or anticyclic citrullinated peptide antibody (CCP) and/or anticyclic citrullinated peptide antibody 2 (CCP2) status.

In order to stratify trials according to the DMARD history of their included patients, we will ask the following signalling question: had the participants, prior to inclusion, potentially exhausted the treatment potential of at least one class of DMARD—either csDMARDs, bDMARD or tsDMARD)?

- csDMARD-naïve (patients were either csDMARD naïve or had not exhausted the treatment potential of at least one csDMARD).

- csDMARD-IR (csDMARD-inadequate responders (IRs)), where patients had exhausted at least one csDMARD option previously.

- DDMARD or tsDMARD-IR (bDMARD or tsDMARD-IRs), where patients had inadequate response to at least one previous bDMARD or tsDMARD. Inclusion in this group entails exclusion from other DMARD history groups.

Patients' concomitant medication (background) of DMARDs during the trial period will be ordered in the following three levels

- MTX.

- csDMARDs other than MTX.

- bDMARDs or tsDMARDs.

We will extract information about how the three groups were handled at randomisation, potentially enabling us to cluster the trials into one of the following five levels: (1) naïve (ie, patients had never used the $\operatorname{drug}(\mathrm{s})$ of interest); (2) not using (ie, the study included only patients who were currently not using the $\operatorname{drug}(\mathrm{s})$ of interest); (3) discontinued (ie, patients were not allowed to continue drug (s) of interest; (4) continued (ie, patients were allowed to continue $\operatorname{drug}(\mathrm{s})$ of interest); and (5) not reported (unclear: no information was reported on this matter). In the case of axSpa, non-steroidal antiinflammatory drugs will be extracted and considered equivalent to csDMARDs.

Data on the following aggregate (average/median) patient baseline characteristics will be extracted

Age, female (proportion), disease duration (years), ESR (mm/hour), CRP (mg/dL), Disease Activity Score, RF positive (proportion), anti-CCP positive (proportion), anti-CCP2 positive (proportion), SJC, TJC, Health Assessment Questionnaire-Disability Index, physician global assessment (eg, Visual Analogue Scale (VAS)), patient global assessment (eg, VAS), and patient-reported pain on VAS of $0-100 \mathrm{~mm}$ (eg, VAS).

\section{RoB and trial characteristics in individual studies}

Many published reviews include some form of RoB assessment (ie, at least for the most frequently used RoB domains) for each individual trial; these assessments will be mapped to the best of our ability to correspond to the Cochrane RoB V.1. ${ }^{35}$ The RoB will also be assessed by one of the two reviewers (JB or EM) and subsequently 
compared with the original bias and internal validity assessments in the review, from which it was sampled.

Within each full-text trial report, we will apply the following domains of the original Cochrane RoB tool (ie, RoB V.1.0), ${ }^{35}$ which comprise methods for:

- Sequence generation/maintaining allocation concealment (selection bias).

- Blinding both patients and personnel (performance bias).

- Management/analysis/reporting of incomplete outcome data (attrition bias).

Each of these three domains will be rated as high risk, low risk or unclear RoB. ${ }^{35}$ In case of uncertainty, another reviewer (SMN/RC) will be consulted. Any discrepancy between RoB assessment sources will be resolved by discussion among the authors.

The following additional bias sources will also be assessed, and data will be extracted from them:

- Single versus multisite trials. ${ }^{36}$

- Small versus large trials (where small will pragmatically be defined as $<100$ patients per arm).${ }^{3738}$

- Source of funding grouped into one of the following five categories: (1) 100\% industry (pharmaceutical/ device company) funded; (2) mixed funding (eg, non-industry and industry); (3) provision of drug only; (4) $100 \%$ non-profit funded; and (5) unclear/ undisclosed funding. ${ }^{39}$

\section{Outcome measures}

Data extraction for each RCT will include the number of patients who died and the numbers of patients with any of the following 'generic events': (1) all WDs, (2) WDs due to AEs and (3) SAEs. ${ }^{17}$ If the number of individual patients with SAEs is not reported, we will extract the number of reported SAEs instead.

Where reasons are provided to explain the SAEs, they will be categorised according to MedDRA V.23.0: (1) infections and infestations; (2) neoplasms benign, malignant and unspecified (including cysts and polyps); (3) cardiac and vascular disorders; or (4) others. If patients in the comparison group later are offered the intervention (ie, switching to the intervention group), the number of events before the switch will be used as the endpoint of choice (ie, before introducing adaptive trial designs).

\section{Summary measures}

For each binary outcome, we will extract data corresponding to a $2 \times 2$ table, summarising the number of patients who experienced the outcome in each comparison group as reported in the randomised trial and the total number of patients randomly assigned in each group. For outcomes collected and reported corresponding to different time points, we will select the time point with the longest follow-up while still respecting the primary research design (eg, before introducing an adaptive trial design, and/or open label extension). ${ }^{4041}$

Because all the outcome measures are dichotomous, the relative risk ratios (RRs) with $95 \%$ CIs will be the preferred measure of relative effect, and these can subsequently be applied to the baseline or control group risks to generate absolute risks. ${ }^{42}$ When appropriate for the very rare adverse events, we will use a continuity correction (adding to all cells a factor proportional to the reciprocal of the size of the contrasting study group), as suggested by Sweeting and colleagues, ${ }^{43}$ to take into account zero cell counts in one group only. This continuity correction will be applied when no events are observed in one study arm of a trial. The correction is inversely proportional to the relative size of the opposite of the study. ${ }^{21}{ }^{43}$ Continuity correction for the experimental intervention arm is $1 /(R+1)$; $R$ is the ratio of control group to intervention group sizes (ie, $R=n_{\mathrm{C}} / n_{\mathrm{I}}$ ). Similarly, the continuity correction for the control comparator arm is $R /(R+1)$. We expect that most trials will report only a few SAEs and deaths, so the ORs and 95\% CIs will also (for the purpose of sensitivity analysis) be calculated with the use of the Peto method. Because the expected events are sparse and all trials will have similar durations of follow-up for their treatment groups, the use of both RRs and ORs should represent a valid approach to assessing the 'risk' associated with the use of synthetic or biological intervention in patients with IA. The number of studies to be included will be determined by the number of eligible trials available in the systematic reviews; hence, a formal sample size calculation would not be meaningful. However, we expect the search to bring around 175 eligible trials (RA: 117, PsA: 15, AxSpA: 43) based on a few large systematic reviews within the field. ${ }^{44-48}$

\section{Synthesis of results}

Outcome events will be coded so that an RR direction of more than one indicates a potentially harmful effect of the experimental intervention (ie, $\mathbf{R R}=\left[r_{\mathrm{I}} / n_{\mathrm{I}}\right] /\left[r_{\mathrm{C}} / n_{\mathrm{C}}\right]$ ). Mixed effects restricted maximum likelihood (REML) meta-analyses ${ }^{49}$ will be used to combine the harm effects across RCTs (based on their $\log (\mathrm{RR}))^{50}$; we will apply the trial ID as a random effect while applying the review ID (from which the trial was sampled) as a fixed effect in order to model the hierarchical structure of the data sampling. ${ }^{51}$

Heterogeneity across randomised comparisons will be assessed by using the Cochrane $Q$ test,${ }^{52}$ interpreted based on the $\mathrm{I}^{2}$ inconsistency index,${ }^{53}$ and quantified by the estimate for between-trial variance $\left(\tau^{2}\right)$, estimated as $T^{2}{ }^{54}$ The effect of population and trial characteristics (listed further) on the between-trial variance will be calculated by univariably adding a fixed factor for the specific characteristic in the model. If introducing a specific covariate into the metamodel reduces the observed between-trial variance $\left(T^{2}\right)$, this result will be considered an indication of a potentially important effect modifier. ${ }^{55}$

Further analyses might (if possible) include multivariable models. ${ }^{56}$ Observational by nature, metaepidemiological studies like the present should be expected to have some degree of metaconfounding because prognostic factors might be unequally distributed between 
studies exposed (trial characteristic positive) or not. We will attempt to adjust for possible confounding covariates (ie, 'deconfound') the inference; that is, we will explore the ability to adjust for any important pre-exposure covariate $C$ (ie, something that happened before the 'study design variable of interest was generated') that is potentially unequally distributed between the metaepidemiological exposure groups (E: exposed vs unexposed), which is also an ancestor (ie, likely cause) of the trial outcome (ie, a more exaggerated risk of harm): $M_{\mathrm{E}} \leftarrow \mathrm{C} \rightarrow Y_{\mathrm{E}^{*}}{ }^{56}$

\section{Power and sample size considerations}

Although the number of trials being eligible in the synthesis is fixed based on the premises, there are several factors that can influence the statistical power in metaanalyses, such as the total number of eligible RCTs $(k)$, their individual sample sizes $\left(N_{1}, N_{2}, \ldots, N_{\mathrm{k}}\right)$ and the number of harm events observed in each of the trials $\left(e_{1}, e_{2}, \ldots, e_{\mathrm{k}}\right)$. Since we want to study harmful effects of various experimental interventions, even very small effect sizes would be informative. Substantial heterogeneitybetween exposed versus unexposed subgroups-will affect the precision of our meta-analytic estimates, and thus our potential to find significant differences between strata. Thus, on an ad hoc basis, we performed some analyses exploring how large the difference between two strata had to be in order for us to be able to detect it, given the expected number of studies at our disposal (say, at least 200 trials across conditions). ${ }^{8} 295758$ An ad hoc subgroup power analysis was conducted in $\mathrm{R}$ using the power.analysis.subgroup function, based on the approach described by Hedges and Pigott: ${ }^{59}$ assuming that we want to compare two independent $\mathrm{ORs}, \mathrm{OR}_{1}=2$ vs $\mathrm{OR}_{2}=1$, with a conservative guestimate of the SE of the $\log (\mathrm{OR})$ values of $0.15,{ }^{61}$ we would have a good statistical power to detect a difference between strata $(90.4 \%)$. The output also tells us that, all else being equal, the effect size difference $\left(\log \left(\mathrm{OR}_{1}\right)\right.$ vs $\left.\log \left(\mathrm{OR}_{2}\right)\right)$ needs to be at least 0.595 in order to reach sufficient power.

\section{Box 3 Risk of bias}

\section{Cochrane risk of bias domains \\ Risk of selection bias. \\ Risk of performance bias. \\ - Risk of attrition bias. \\ - Overall risk of bias.*}

\section{Additional bias sources}

S Single versus multisite trials.

Small versus large trials.

- Source of funding.

*For each trial, the overall RoB will be classified as low (ie, low RoB for all three domains), high (ie, high RoB for one or more domains) or unclear (ie, unclear $\mathrm{RoB}$ for one or more domains in the absence of high RoB).

RoB, risk of bias.

\section{RoB across studies}

Stratified meta-analyses will be used for tests of interaction between harms and the trial RoB (listed in box 3) and trial characteristics collected as described previously.

\section{DISCUSSION}

The Outcome Measures in Rheumatology initiative established the Contextual Factors Working Group to guide the understanding, identification and handling of contextual factors for clinical trials, with most of the current emphasis being on net benefit inferred from a rheumatology trial. ${ }^{62}$ This metaepidemiological study will work from the original definition of what constitutes a contextual factor, as defined in the introduction. We will explore and hope to reveal the possible impact of contextual factors (ie, population and trial characteristics) and RoB domains of three different (but related) harm measures, as well as deaths. ${ }^{63}$ Knowing which factors are associated with a causal model for harms-either as effect modifiers or distortions of the outcome due to bias-is important for improving investigation and reporting of harms in future trials. ${ }^{64}$ If future trials report harms according to important contextual factors, meta-analyses would be able to investigate contextual factors for harms without relying on getting access to individual patient data. Such metaresearch might identify subgroups among patients with rheumatic disease that are at higher risk of experiencing harms. Such information would provide important evidence for future treatment guideline development. Ultimately, the doctor will be able to differentiate the risk of intervention based on the patient's characteristics. This has the potential to enhance informed decision-making and effect therapeutic interventions applied in practice, leading to safer treatment of individual patients and increase efficiency in the healthcare system.

This study has several strengths. This will be a comprehensive risk of harm analysis of bDMARDs and tsDMARDs across multiple IA diagnoses unlike previous assessments. The study selection, data extraction and RoB assessment will be done by two reviewers; the data extraction will be extensive as it will include data on many different contextual factors and RoB items. Finally, we will only include RCTs to avoid inherent problems when investigating potential effect modifiers from non-RCT evidence.

Nevertheless, several limitations should be considered. First, the analyses may be limited by poor reporting, and data are likely to be lacking for certain contextual factors (ie, exposures) and uncommon harms (ie, outcomes). Second, by relying on the search results of published reviews, we assume that we retrieve a representative sample for our analysis; however, it is possible that potentially useful trials may be omitted, for example, due to very specific eligibility criteria in the reviews or very recent trials that have not yet been included in a systematic review, which could theoretically influence our results. Third, other characteristics that we do not consider may be relevant. Fourth, metaregression assessments, 
despite including only RCTs, are observational by nature and their results may by themselves be confounded by other factors. ${ }^{65}$ Finally, the metaregression assessments involving aggregated data may be affected by ecological fallacy and could potentially be misleading. ${ }^{66}$

\section{Patient and public involvement}

Patients and/or the public were not involved in designing study concept or drafting of the protocol.

\section{ETHICS AND DISSEMINATION}

Because our study does not collect primary data, no formal ethical assessment and informed consent are required. The study will be disseminated in a peerreviewed publication.

\section{Author affiliations}

${ }^{1}$ Section for Biostatistics and Evidence-Based Research, the Parker Institute, Bispebjerg and Frederiksberg Hospital, Copenhagen, Denmark

${ }^{2}$ Research Unit of Rheumatology, Department of Clinical Research, University of Southern Denmark, Odense University Hospital, Odense, Denmark

${ }^{3} 3$ Department of Medicine, Department of Epidemiology and Population Health, Department of Biomedical Data Science, Department of Statistics, and MetaResearch Innovation Center at Stanford (METRICS), Stanford University, Stanford, CA, USA

${ }^{4}$ Division of Rheumatology, Department of Medicine, University of California at Los Angeles, LA, CA, USA

${ }^{5}$ Division of Rheumatology, Department of Medicine, Medical University of Vienna, Vienna, Austria

${ }^{6}$ Nuffield Department of Orthopaedics, Rheumatology and Musculoskeletal

Sciences, University of Oxford, Oxford, UK

Acknowledgements The Parker Institute is grateful for the financial support received from public and private foundations, companies and private individuals over the years.

Contributors Contributions: study concept and design and drafting of the protocol: EM, JB, SMN, TE, LEK, RC, JPAI, ST, DF, JSS and PCT; critical revision of the protoco for important intellectual content and final approval before submission: all authors; guarantor of the protocol: RC; obtained funding: TE and RC.

Funding The Parker Institute, Bispebjerg and Frederiksberg Hospital, is supported by a core grant from the Oak Foundation (OCAY-18-774-OFIL). The Oak Foundation is a group of philanthropic organisations that, since its establishment in 1983, has given grants to not-for-profit organisations around the world.

Disclaimer The views expressed in the submitted protocol are the authors' own and not an official position of the institution or funder.

Competing interests None declared.

Patient and public involvement Patients and/or the public were not involved in the design, conduct, reporting or dissemination plans of this research.

Patient consent for publication Not required.

Provenance and peer review Not commissioned; externally peer reviewed.

Open access This is an open access article distributed in accordance with the Creative Commons Attribution Non Commercial (CC BY-NC 4.0) license, which permits others to distribute, remix, adapt, build upon this work non-commercially, and license their derivative works on different terms, provided the original work is properly cited, appropriate credit is given, any changes made indicated, and the use is non-commercial. See: http://creativecommons.org/licenses/by-nc/4.0/.

\section{ORCID iDs}

Esben Malm http://orcid.org/0000-0002-8016-6646

Sabrina Mai Nielsen http://orcid.org/0000-0003-2857-2484

John P. A. loannidis http://orcid.org/0000-0003-3118-6859

Peter C. Taylor http://orcid.org/0000-0001-7766-6167

Torkell Ellingsen http://orcid.org/0000-0003-0426-4962

\section{REFERENCES}

1 Ledingham J, Snowden N, Ide Z. Diagnosis and early management of inflammatory arthritis. BMJ 2017;358:j3248.

2 Raychaudhuri SP, Deodhar A. The classification and diagnostic criteria of ankylosing spondylitis. J Autoimmun 2014;48-49:128-33.

3 Miyabe Y, Miyabe C, Luster AD. LTB and BLT1 in inflammatory arthritis. Semin Immunol 2017;33:52-7.

4 Mclnnes IB, Schett $\mathrm{G}$. The pathogenesis of rheumatoid arthritis. $N$ Engl J Med 2011;365:2205-19.

5 Janke K, Biester K, Krause D, et al. Comparative effectiveness of biological medicines in rheumatoid arthritis: systematic review and network meta-analysis including aggregate results from reanalysed individual patient data. BMJ 2020;370:m2288.

6 Schweikert B, Malmberg C, Núñez M, et al. Cost-effectiveness analysis of ixekizumab versus secukinumab in patients with psoriatic arthritis and concomitant moderate-to-severe psoriasis in Spain. BMJ Open 2020;10:e032552.

7 Hartman L, Rasch LA, Klausch T, et al. Harm, benefit and costs associated with low-dose glucocorticoids added to the treatment strategies for rheumatoid arthritis in elderly patients (GLORIA trial): study protocol for a randomised controlled trial. Trials 2018;19:67.

8 Christensen AW, Tarp S, Furst DE, et al. Most trial eligibility criteria and patient baseline characteristics do not modify treatment effect in trials using targeted therapies for rheumatoid arthritis: a MetaEpidemiological study. PLoS One 2015;10:e0136982.

9 Callhoff J, Sieper J, Weiß A, et al. Efficacy of TNF $\alpha$ blockers in patients with ankylosing spondylitis and non-radiographic axial spondyloarthritis: a meta-analysis. Ann Rheum Dis 2015;74:1241-8.

10 Goulabchand R, Mouterde G, Barnetche T, et al. Effect of tumour necrosis factor blockers on radiographic progression of psoriatic arthritis: a systematic review and meta-analysis of randomised controlled trials. Ann Rheum Dis 2014;73:414-9.

11 Oo C, Kalbag SS. Leveraging the attributes of biologics and small molecules, and releasing the bottlenecks: a new wave of revolution in drug development. Expert Rev Clin Pharmacol 2016;9:747-9.

12 Komaki Y, Yamada A, Komaki F, et al. Efficacy, safety and pharmacokinetics of biosimilars of anti-tumor necrosis factor- $\alpha$ agents in rheumatic diseases; a systematic review and metaanalysis. J Autoimmun 2017;79:4-16.

13 So A, Inman RD. An overview of biologic disease-modifying antirheumatic drugs in axial spondyloarthritis and psoriatic arthritis. Best Pract Res Clin Rheumatol 2018;32:453-71.

14 Gadina M, Le MT, Schwartz DM, et al. Janus kinases to jakinibs: from basic insights to clinical practice. Rheumatology 2019;58:i4-16.

15 Sarzi-Puttini P, Ceribelli A, Marotto D, et al. Systemic rheumatic diseases: from biological agents to small molecules. Autoimmun Rev 2019;18:583-92.

16 Winthrop KL. The emerging safety profile of JAK inhibitors in rheumatic disease. Nat Rev Rheumatol 2017;13:234-43.

17 loannidis JPA, Evans SJW, Gøtzsche PC, et al. Better reporting of harms in randomized trials: an extension of the CONSORT statement. Ann Intern Med 2004;141:781-8.

18 Singh JA, Wells GA, Christensen R, et al. Adverse effects of biologics: a network meta-analysis and Cochrane overview. Cochrane Database Syst Rev 2011;2:Cd008794.

19 Minozzi S, Bonovas S, Lytras T, et al. Risk of infections using antiTNF agents in rheumatoid arthritis, psoriatic arthritis, and ankylosing spondylitis: a systematic review and meta-analysis. Expert Opin Drug Saf 2016;15:11-34.

20 Grøn KL, Arkema EV, Glintborg B, et al. Risk of serious infections in patients with rheumatoid arthritis treated in routine care with abatacept, rituximab and tocilizumab in Denmark and Sweden. Ann Rheum Dis 2019;78:320-7.

21 Bongartz T, Sutton AJ, Sweeting MJ, et al. Anti-TNF antibody therapy in rheumatoid arthritis and the risk of serious infections and malignancies: systematic review and meta-analysis of rare harmful effects in randomized controlled trials. JAMA 2006;295:2275-85.

22 Salgado E, Maneiro JR, Carmona L, et al. Safety profile of protein kinase inhibitors in rheumatoid arthritis: systematic review and metaanalysis. Ann Rheum Dis 2014;73:871-82.

23 Sepriano A, Kerschbaumer A, Smolen JS, et al. Safety of synthetic and biological DMARDs: a systematic literature review Informing the 2019 update of the EULAR recommendations for the management of rheumatoid arthritis. Ann Rheum Dis 2020;79:760-70.

24 Mease P, Charles-Schoeman C, Cohen S, et al. Incidence of venous and arterial thromboembolic events reported in the tofacitinib rheumatoid arthritis, psoriasis and psoriatic arthritis development programmes and from real-world data. Ann Rheum Dis 2020;79:1400-13.

25 Rahman MU, Buchanan J, Doyle MK, et al. Changes in patient characteristics in anti-tumour necrosis factor clinical trials for 
rheumatoid arthritis: results of an analysis of the literature over the past 16 years. Ann Rheum Dis 2011;70:1631-40.

26 Gluud LL. Bias in clinical intervention research. Am J Epidemiol 2006:163:493-501.

27 Boers M, Kirwan JR, Wells G, et al. Developing core outcome measurement sets for clinical trials: OMERACT filter 2.0. J Clin Epidemiol 2014;67:745-53.

28 Savović J, Jones HE, Altman DG, et al. Influence of reported study design characteristics on intervention effect estimates from randomized, controlled trials. Ann Intern Med 2012;157:429-38.

29 Ballegaard C, Jørgensen TS, Skougaard M, et al. Trial characteristics as contextual factors when evaluating targeted therapies in patients with psoriatic disease: a Meta-Epidemiologic study. Arthritis Care Res 2018;70:1206-17.

30 Bae J-M. Meta-epidemiology. Epidemiol Health 2014;36:e2014019.

31 Bolvig J, Juhl CB, Boutron I, et al. Some Cochrane risk-of-bias items are not important in osteoarthritis trials: a meta-epidemiological study based on Cochrane reviews. J Clin Epidemiol 2018;95:128-36.

32 Dossing A, Tarp S, Furst DE, et al. Modified intention-to-treat analysis did not bias trial results. J Clin Epidemiol 2016;72:66-74.

33 Higgins JPT LT, Chandler J, Tovey D, et al. Methodological expectations of Cochrane intervention reviews. London: Cochrane, 2018: Version 1.06

34 Shamseer L, Moher D, Clarke M, et al. Preferred reporting items for systematic review and meta-analysis protocols (PRISMA-P) 2015: elaboration and explanation. BMJ 2015;350:97647.

35 Higgins JPT, Altman DG, Gøtzsche PC, et al. The Cochrane collaboration's tool for assessing risk of bias in randomised trials. BMJ 2011;343:d5928.

36 Dechartres A, Boutron I, Trinquart L, et al. Single-center trials show larger treatment effects than multicenter trials: evidence from a metaepidemiologic study. Ann Intern Med 2011;155:39-51.

37 Nüesch E, Trelle S, Reichenbach S, et al. Small study effects in metaanalyses of osteoarthritis trials: meta-epidemiological study. BMJ 2010;341:c3515.

38 Kjaergard LL, Villumsen J, Gluud C. Reported methodologic quality and discrepancies between large and small randomized trials in meta-analyses. Ann Intern Med 2001;135:982-9.

39 Lundh A, Lexchin J, Mintzes B, et al. Industry sponsorship and research outcome. Cochrane Database Syst Rev 2017;2:Mr000033.

40 Pickles T, Alten R, Boers M, et al. Adaptive trial designs in rheumatology: report from the OMERACT special interest group. $J$ Rheumatol 2019;46:1406-8

41 Buch MH, Silva-Fernandez L, Carmona L, et al. Development of EULAR recommendations for the reporting of clinical trial extension studies in rheumatology. Ann Rheum Dis 2015;74:963-9.

42 Guyatt GH, Oxman AD, Santesso N, et al. GRADE guidelines: 12. preparing summary of findings tables-binary outcomes. J Clin Epidemiol 2013;66:158-72.

43 Sweeting MJ, Sutton AJ, Lambert PC. What to add to nothing? Use and avoidance of continuity corrections in meta-analysis of sparse data. Stat Med 2004;23:1351-75.

44 Singh JA, Hossain A, Tanjong Ghogomu E, et al. Biologics or tofacitinib for people with rheumatoid arthritis unsuccessfully treated with biologics: a systematic review and network meta-analysis. Cochrane Database Syst Rev 2017;3:CD012591.

45 Singh JA, Hossain A, Mudano AS, et al. Biologics or tofacitinib for people with rheumatoid arthritis naive to methotrexate: a systematic review and network meta-analysis. Cochrane Database Syst Rev 2017;5:CD012657.

46 Singh JA, Hossain A, Tanjong Ghogomu E, et al. Biologic or tofacitinib monotherapy for rheumatoid arthritis in people with traditional disease-modifying anti-rheumatic drug (DMARD) failure: a Cochrane systematic review and network meta-analysis (NMa). Cochrane Database Syst Rev 2016;11:CD012437.

47 Fidahic M, Jelicic Kadic A, Radic M. Celecoxib for rheumatoid arthritis. Cochrane Database Syst Rev 2017;6:CD012095.

48 Lawson DO, Eraso M, Mbuagbaw L, et al. Tumor necrosis factor inhibitor dose reduction for axial spondyloarthritis: a systematic review and Meta-Analysis of randomized controlled trials. Arthritis Care Res 2021;73:861-72.

49 Normand SL. Meta-analysis: formulating, evaluating, combining, and reporting. Stat Med 1999;18:321-59.

50 van Houwelingen HC, Arends LR, Stijnen T. Advanced methods in meta-analysis: multivariate approach and meta-regression. Stat Med 2002;21:589-624.

51 Sterne JA, Jüni P, Schulz KF, et al. Statistical methods for assessing the influence of study characteristics on treatment effects in 'metaepidemiological' research. Stat Med 2002;21:1513-24.

52 Cochran WG. The combination of estimates from different experiments. Biometrics 1954:10:101-29.

53 Higgins JPT, Thompson SG, Deeks JJ, et al. Measuring inconsistency in meta-analyses. BMJ 2003;327:557-60.

54 Thompson SG, Higgins JPT. How should meta-regression analyses be undertaken and interpreted? Stat Med 2002;21:1559-73.

55 Sterne JAC, Jüni P, Schulz KF, et al. Statistical methods for assessing the influence of study characteristics on treatment effects in 'metaepidemiological' research. Stat Med 2002;21:1513-24.

56 Christensen R, Berthelsen DB. Controversy and debate on Metaepidemiology. paper 3: causal inference from meta-epidemiology: a reasonable goal, or wishful thinking? $J$ Clin Epidemiol 2020;123:131-2.

57 Tarp S, Eric Furst D, Boers M, et al. Risk of serious adverse effects of biological and targeted drugs in patients with rheumatoid arthritis: a systematic review meta-analysis. Rheumatology 2017;56:417-25.

58 Andreasen RA, Kristensen LE, Baraliakos X, et al. Assessing the effect of interventions for axial spondyloarthritis according to the endorsed ASAS/OMERACT core outcome set: a meta-research study of trials included in Cochrane reviews. Arthritis Res Ther 2020;22:177.

59 Hedges LV, Pigott TD. The power of statistical tests for moderators in meta-analysis. Psychol Methods 2004;9:426-45.

60 Hedges LV, Pigott TD. The power of statistical tests in meta-analysis. Psychol Methods 2001;6:203-17.

61 Singh JA, Cameron C, Noorbaloochi S, et al. Risk of serious infection in biological treatment of patients with rheumatoid arthritis: a systematic review and meta-analysis. Lancet 2015;386:258-65.

62 Nielsen SM, Uggen Rasmussen M, Boers M, et al. Towards consensus in defining and handling contextual factors within rheumatology trials: an initial qualitative study from an OMERACT Working group. Ann Rheum Dis 2021;80:242-9.

63 Nielsen SM, Storgaard H, Ellingsen T, et al. Population characteristics as important contextual factors in rheumatological trials: an exploratory meta-epidemiological study from an OMERACT Working group. Ann Rheum Dis 2020;79:1269-76.

64 Finger ME, Boonen A, Woodworth TG, et al. An OMERACT initiative toward consensus to identify and characterize candidate contextual factors: report from the contextual factors Working group. $J$ Rheumatol 2017:44:1734-9.

65 Thompson SG, Higgins JPT. Treating individuals 4: can meta-analysis help target interventions at individuals most likely to benefit? Lancet 2005;365:341-6

66 Berlin JA, Santanna J, Schmid CH, et al. Individual patient- versus group-level data meta-regressions for the investigation of treatment effect modifiers: ecological bias rears its ugly head. Stat Med 2002;21:371-87. 